\title{
The Ultrastructure of Hybrid Acute Leukemia: A Study of 15 Cases
}

Yong-xin Ru, MD, Hui-jun Wang, MD, Bing-xin Yang, BSc, Jin-hua Liu, BSc, Zhan-qi $\mathrm{Li}, \mathrm{BSC}$, Cheng-wen Li, BSC, Jian-xiang Wang, MD, and Ying-chang Mi, MD

Institute of Hematology \& Blood Diseases Hospital, Peking Union College, Tianjin, China
Received 25 February 2005; accepted 12 April 2005.

We thank B. Eyden (clinic scientist in the Department of Histopathology, Christie Hospital NHS, Manchester, UK) for revising this paper. We also thank hematologists in our hospital for providing the cases studied.

Address correspondence to Dr. Yong-xin Ru, Department of Electron Microscopy, Institute of Hematology \& Blood Diseases Hospital, Peking Union College, Tianjin, 300020, China. E-mail: ruyongxin@.tom.com

\begin{abstract}
The objective of this study was to investigate the ultrastructural characteristics of hybrid acute leukemia (HAL). Fifteen cases of HAL were studied by transmission electron microscopy (TEM), focusing on organelles and myeloperoxidase (MPO) reaction of leukemic cells. By TEM, 5 out 15 cases of HAL were consistent with immunophenotyping ( 3 cases of biphenotypic type, and 2 cases of biclonal type with granulocytes and lymphocytes); 2 cases were suspected as HAL. On other hand, 5 cases of HAL were assigned to ALL, and 2 cases were misinterpreted as M5a and 1 as M4b. Most of the blast cells of biphenotypic HAL showed lymphoid features, except some cases containing MPO positive granules in blasts, while a few cases exhibited monocytic or nonspecific features. TEM offers advantages in the diagnosis of biclonal type HAL and biphenotypic HAL positive for MPO. However, it is difficult to differentiate MPO-negative cases of biphenotypic HAL from ALL and a few cases may be misinterpreted as M5 by TEM.
\end{abstract}

KEYWORDS diagnosis, hybrid acute leukemia, ultrastructure

Hybrid acute leukemia (HAL) is a rare disease with dismal prognosis. It was called acute myelocytic and lymphocytic leukemia (AMLL) collectively in the early literature, because the blasts maintained varied immunophenotypic profiles from multiple lineage antigens in individual patients. It is called biphenotypic type because individual neoplastic cells express both of myeloid and lymphoid antigens simultaneously, but biclonal type because there is both myeloid and lymphoid malignant cell population in a patient [1]. The cell components and structure of HAL are poorly documented by transmission electron microscope (TEM) because of the rarity and variety in the condition. The present paper documented the ultrastructural features of HAL in 15 cases diagnosed by flow cytometry.

\section{MATERIALS AND METHODS Clinical Materials}

The study consisted of 15 cases of untreated HAL, who were accessions of Blood Diseases Hospital from 2003 to 2004 (Table 1). The primary diagnoses were supposed from the outpatient department in terms of morphological examination of bone marrow slides. Five of 15 cases were classified in acute 
TABLE 1 Clinical Data of 15 Cases with HAL

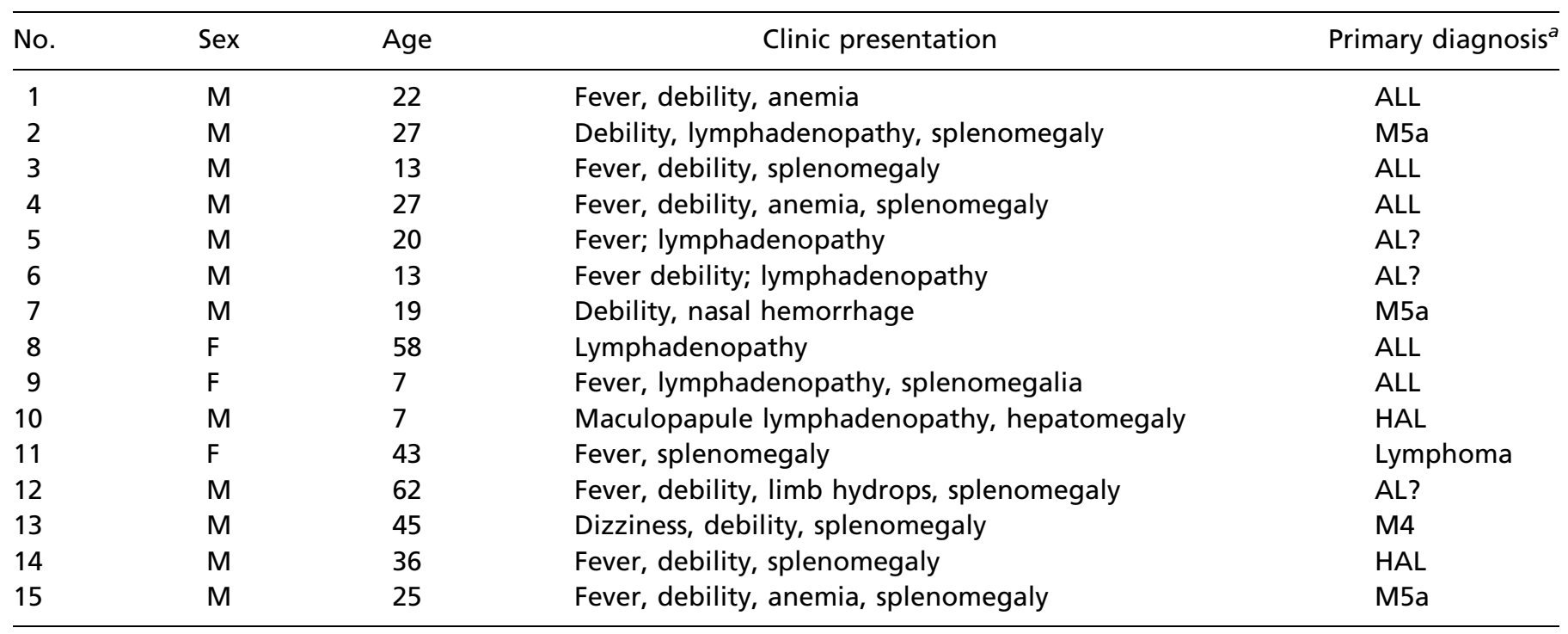

${ }^{a}$ According to morphology of bone marrow slides in outpatient department.

lymphoblastic leukemia (ALL), 3 in acute monocytic leukemia (AMOL). Two cases were suspected to be HAL, 1 to be acute myeloid monocytic leukemia (M4), and 1 to be lymphoma; and for 3 there were no diagnostic suggestions. The chromosome analysis were carried out in 10 patients, 4 of which were involved the Philadelphia ( $\mathrm{Ph}$ ) translocation (3 cases were $\mathrm{t}(9,22)$ ( $\mathrm{q} 34 ; \mathrm{q} 11) ; 1$ case had no cytogenetic report, but was positive for $\mathrm{BCR} / \mathrm{ABL}$ ), 5 with normal karyotype, 3 with other structural abnormalities of chromosome (Table 2). Meanwhile, all of blood cells from their bone marrow were measured by flow cytometry and electron microscopy simultaneously. The final diagnosis of HAL was made on the basis of the European group for the immunological characterization of leukemias and clinical features [2, 3].

\section{Flow Cytometry Analysis}

Heparinized samples of bone marrow and peripheral blood were prepared for flow cytometric analysis [4]. Immunophenotyping staining procedures were standard whole blood lysis techniques using FACSLyse (BDIS). Cytoplasmic and nuclear antigens were detected by flow cytometry using FACS Permeabilization solution (Becton Dickinson). For 3-color staining, directly conjugated monoclonal antibodies were added: the lymphoid markers were monoclonal antibodies (MoAbs) for CD2, CD3, CD4, CD5, CD7, CD8, CD10, CD19, CD20, CD22, and CD79a, and the myeloid markers were MoAbs against CD13, CD14, CD33, CD33, CD117, CD14, CD16, CD64, and cMPO. CD34 and HLA-DR also were detected. All were from Becton Dickinson. As third fluorescence sources, antibodies conjugated by peridinin chlorophyll A protein (CD45-PerCP) were used. Specimens were analyzed on a FACSCalibur

TABLE 2 Cytogenesis in 15 Patients with HAL

\begin{tabular}{|c|c|c|}
\hline No. & Age/sex & Karyotype \\
\hline 1 & $22 / \mathrm{M}$ & NT \\
\hline 2 & $27 / M$ & $\begin{array}{l}\text { 46, } \mathrm{XY}, \mathrm{t}(9,22)(\mathrm{q} 34 ; \mathrm{q} 11)[5] / 46, \mathrm{XY}[2] \\
52-53, \mathrm{XY},+\mathrm{Y},+2,+6,+8,-9, \mathrm{t}(9,22) \\
(\mathrm{q} 34 ; 11),+14,+16,+\mathrm{ph}[\mathrm{CP} 2]\end{array}$ \\
\hline 3 & $13 / \mathrm{M}$ & $\begin{array}{l}\text { 46, XY, t(9;22;13)(q34; q11; q14) [10]/40, } \\
\quad X Y, t(9 ; 22 ; 13), \text { add }(21)(q 22)[4]\end{array}$ \\
\hline 4 & $27 / F$ & NT \\
\hline 5 & $20 / M$ & $46, X Y[15]$ \\
\hline 6 & $13 / \mathrm{M}$ & NT \\
\hline 7 & $19 / \mathrm{M}$ & $46, X Y, t(1 ; 21)(p 34 ; 13 p)[10] ; 46, X Y[2]$ \\
\hline 8 & $58 / F$ & $46, X X[6]$ \\
\hline 9 & $7 / F$ & $46, X Y[5]$ \\
\hline 10 & $7 / \mathrm{M}$ & $47, X Y,+11[15]$ \\
\hline 11 & $43 / F$ & $46, X X[9]$ \\
\hline 12 & $62 / M$ & $\begin{array}{l}\text { 46, } X Y, \operatorname{inv}(3)(p 12 ; q 26), t(9,22)(q 34 ; q 11), \\
\text { del }(20)(q 12)[6]\end{array}$ \\
\hline 13 & $45 / \mathrm{M}$ & NT \\
\hline 14 & $36 / \mathrm{M}$ & NT \\
\hline 15 & $25 / M$ & $46, X X[1]$ \\
\hline
\end{tabular}

Note. NT, not tested; Case 14 was positive for BCR/ABL by FISH $(B C R / A B L+)$ 
flow cytometer (Becton Dickinson, San Jose, CA) equipped with Cellquest software (Becton Dickinson). The flow cytometers acquired a minimum of 10,000 cellular events as list mode files containing forward angle scatter, log side scatter, and 3 log fluorescence signals. Files were analyzed using Cellquest software and CD $45 /$ side scatter gating methods. A positive marker was defined as expression on $20 \%$ of blasts for surface antigens, and $10 \%$ of blasts for cytoplasmic and nuclear antigens.

\section{Electron Microscopy}

The bone marrow aspirates for routine electron microscopy were processed as previously described [5]. Briefly, nucleated cells $\left(\times 10^{6}\right.$ cells $)$ isolated from $3-5 \mathrm{~mL}$ of anticoagulated bone marrow aspiration were fixed in $2 \%$ glutaraldehyde in PBS ( $\mathrm{pH} 7.4)$ for $1 \mathrm{~h}$, washed in PBS, postfixed in $1 \%$ osmium tetroxide, dehydrated in graded alcohol, and embedded in Epon 812. The ultrathin sections were stained with uranyl acetate and lead citrate, then they were examined with a Hitachi electron microscope (H-600). For detection of myeloperoxidase (MPO) activities, the method of Roels et al. [6] was used: the cells were incubated for $1 \mathrm{~h}$ in Graham Karnovsky medium [7], fixed in $2 \%$ glutaraldehyde, then processed for electron microscopy as described above, but unstained sections were viewed. MPO-positive cells were first calculated in 200 cells from each specimen, and the percentage of MPO-positive blasts was measured. The case positive for MPO, which was indicated by + , was defined as the positive blasts that surpassed $10 \%$ in calculated blast cells; the negative case of less than $10 \%$ was signed with - . Then the ultrastructural features of blast cells were investigated in routine ultrathin sections.

The studied features included average cell diameter, nucleus-cell ratio and irregularity of nuclear contour, which was estimated semiquantitatively at 3 levels:,+++ , and +++ . The quantity of rough endoplasmic reticulum (RER) and Golgi apparatus was also evaluated by,+++ , and +++ , indicating a little, moderate, and abundant in cytoplasm of blasts. The presence or absence of granules was indicated by + and - , respectively.

\section{RESULTS \\ Immunophenotyping Characteristics (Table 3)}

Thirteen out of 15 cases were biphenotypic type, in which there were 10 cases $(1,2-4,6,9,11-13$, and 15) with coexpression of myeloid and B lymphoid markers, 2 cases ( 5 and 7) with coexpression of myeloid and T-lymphoid markers, and 1 case (8) with T- and B-lymphoid antigens. The other 2 cases (10 and 14) were biclonal type. The antigens of HLADR and CD34 were expressed in all of cases.

TABLE 3 Immunophenotyping Characteristics of 15 Patients with HAL

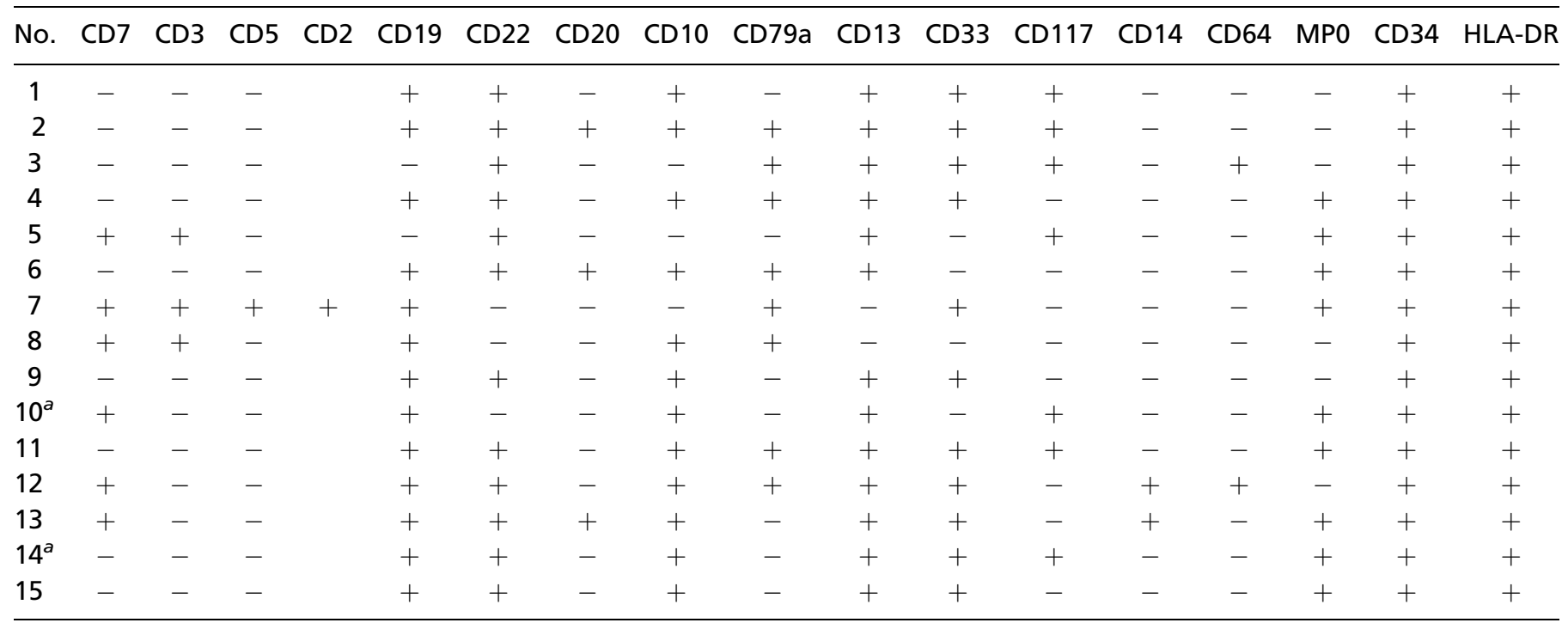

${ }^{a}$ Biclonal type of HAL. 
TABLE 4 Ultrastructural Characteristics and Diagnosis of 15 Patients with HAL

\begin{tabular}{|c|c|c|c|c|c|c|c|c|c|}
\hline No. & $A D(m)$ & MPON/C & $\mathrm{NI}$ & $\mathrm{HC}$ & ER & GB & $\mathrm{Gr}$ & Mit & EMD \\
\hline 1 & 10 & -0.9 & +++ & +++ & ++ & ++ & + & ++++ & ALL \\
\hline 2 & 15 & -0.7 & + & + & + & - & + & ++++ & M5 \\
\hline 4 & 10 & -0.8 & + & +++ & + & + & - & +++ & ALL \\
\hline 5 & 12 & +0.8 & +++ & ++ & +++ & ++ & + & ++++ & HAL \\
\hline 8 & 12 & -0.7 & ++ & ++ & ++ & - & - & +++ & ALL \\
\hline 9 & 11 & -0.7 & + & +++ & +++ & + & + & +++ & M4 \\
\hline \multirow[t]{2}{*}{10} & 11 & -0.7 & ++ & ++++ & ++ & + & - & ++ & $\mathrm{HAL}$ \\
\hline & 13 & +0.6 & +++ & ++ & ++ & ++ & + & ++ & \\
\hline 11 & 13 & +0.6 & ++ & ++ & +++ & ++ & + & ++ & HAL? \\
\hline 15 & 10 & +0.8 & ++ & ++ & + & ++ & + & ++ & $\mathrm{HAL}$ ? \\
\hline
\end{tabular}

Note: $A D$, average diameter; N/C, nucle/cell ratio; NI, nuclear irregularity; HC, heterochromatin; ER, endoplasmic reticulum; GB, Golgi body; Gr, granule; Mit, mitochondria; EMD, electron microscopy diagnosis.

\section{Electron Microscopy Diagnosis and Ultrastructural Features (Table 4)}

On the basis of ultrastructure initially, 5 out of 15 cases were diagnosed as HAL (three of biphenotypic type (3, 5, and 13), 2 of biclonal type (10 and 14)); 2 cases $(11,15)$ were suspected as being biphenotypic. Five cases of biphenotypic type (1, 4, and 6-8) were assigned to ALL, 2 cases of biphenotypic type ( 2 and 12 ) to M5, and 1 case (14) to M4.

All of the blast cells in 5 cases of biphenotypic type explained as ALL by TEM demonstrated lymphoid ultrastructural characteristics, sharing typical round or oval shape with or without short projections on the cell surface. The blast cell diameters ranged from 10 to $14 \mu \mathrm{m}$ and the nucleus-cell ratio generally was 0.8 . The nuclei with prominent nucleoli were usually irregular, exhibiting deep incisures and notches, which frequently contained abundant heterochromatin. In these blast cells, the mitochondria containing disrupted cristae often exhibited an enlarged or swollen image, but fewer cisternae of RER were found under the cell membrane. Abundant free ribosome and patches of glycogen were dispersed or floated in the cytoplasm, and Golgi zones were seldom well developed. They weren't positive cases for MPO and contained few granules on the basis of TEM (Figure 1).
In addition to being positive for MPO, 3 cases of biphenotypic type diagnosed by TEM also shared the partial lymphoid ultrastructural features, including more heterochromatin, higher nucleus-cell ratio, and irregular nuclei, but they often had a plenty of Golgi bodies, more RER, and some granules in the cytoplasm (Figure 2). The 2 cases suspected of being the biphenotypic type of HAL were also positive for

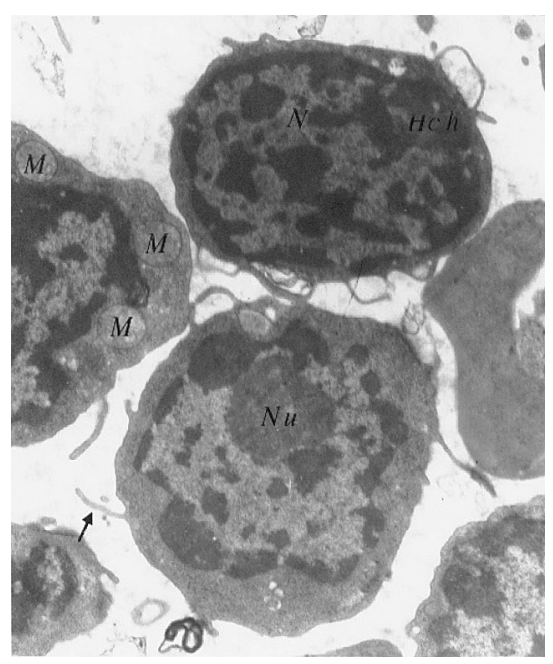

FIGURE 1 Biphenotypic leukemia cells mainly exhibiting lymphoid structure, including cytoplasm processes (arrow), vacuoles, high nucleo-cytoplasmic ratio, increased heterochromatin $(\mathrm{Hch})$, prominent nucleolus, enlarged mitochondria (M). Case 4, $\times 5000$. 


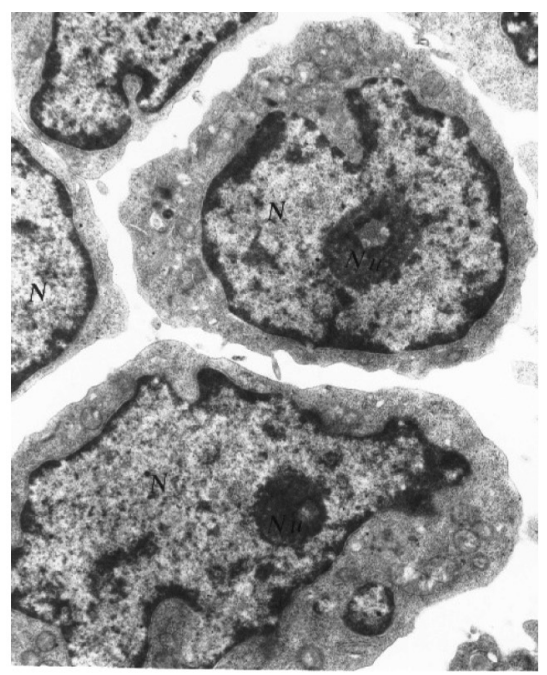

(A)

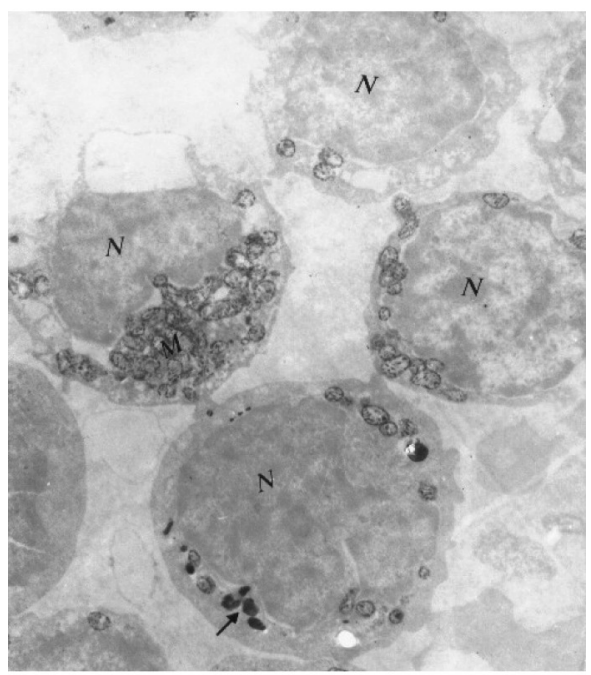

(B)

FIGURE 2 (A) Biphenotypic leukemia cells showing irregular nuclei with deep incisures and notches, prominent nucleolus, dense granules in cytoplasm, $\times \mathbf{5 0 0 0}$; (B) scattered MPO positive granules in cytoplasm (arrow). Case $5, \times 4000$.

MPO; nevertheless, blast cells illustrated typical lymphoid features, including more heterochromatin, less developed Golgi apparatuses, and fewer RER. Two cases of biclonal type were composed of both kinds of blast cells with lymphoid and myeloid ultrastructural features respectively; they were highlighted by MPO examination, Some being positive for MPO, and others negative. The myeloid malignant cells included myeloblasts and premyelocytes that were positive for MPO, and lymphoid blasts had lymphoid features and were negative for MPO (Figure 3). Most blast cells in 2 cases of biphenotypic type diagnosed as M5 by TEM, with shared monocytic

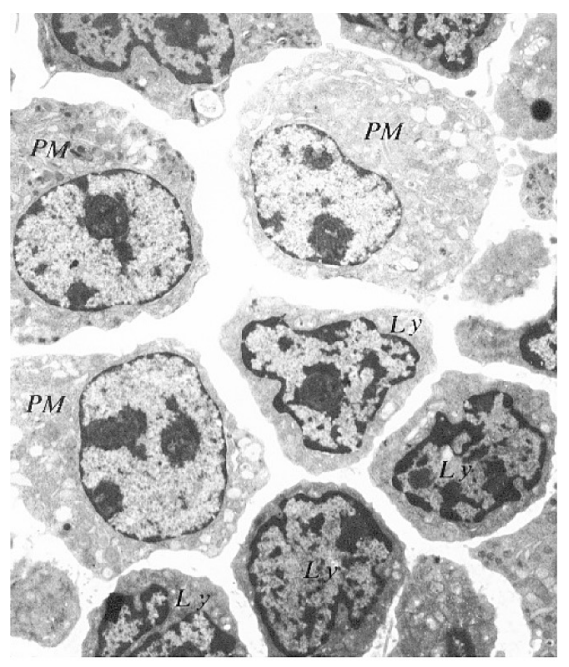

(A)

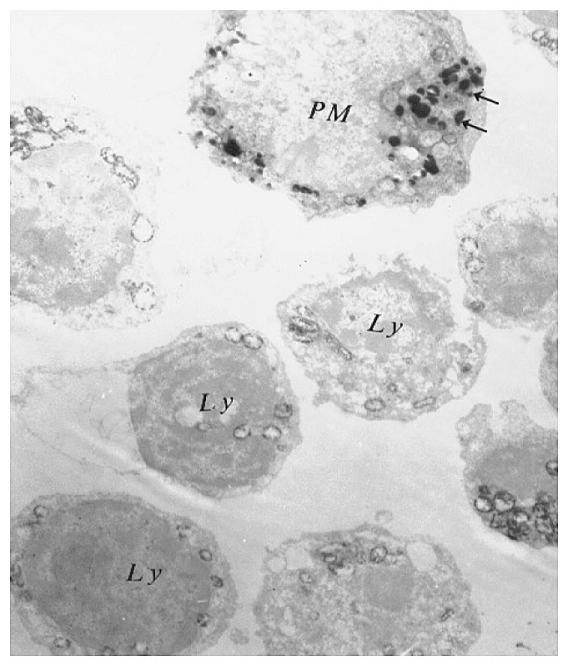

(B)

FIGURE 3 Biclonal type HAL: (A) coexistence of premyelocytic (PM) and lymphoid blasts (Ly), $\times 2500$; (B) premyelocytic blast highlighted by MPO reaction (arrow). Case $14, \times 3000$.

ultrastructural features. The blasts were usually bigger, with diameters between 15 and $16 \mu \mathrm{m}$, and the nucleus-cell ratio was around 0.7. The blast cells always had plenty of cytoplasm and prominent nucleolus in convoluted nuclei that frequent with nuclear pockets and cytoplasmic bridges. The more dense mitochondria were usually small and Golgi bodies were easily found in abundant cytoplasm. They hadn't showed MPO positive under TEM (Figure 4). One case misinterpreted as M4 contained the blast with atypical and heterogeneous ultrastructural features in which the blast was negative for MPO. 


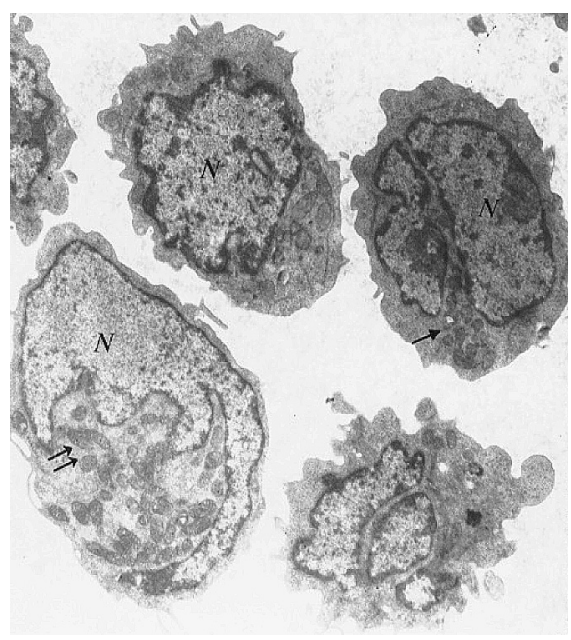

FIGURE 4 Biphenotypic blasts with monocytoid ultrastructural appearances in BAL: large size, plenty of cytoplasm, convoluted bizarre nuclei with prominent nucleolus, small mitochondria with dense matrix (arrow), and Golgi bodies. Case 12, $\times 4000$.

\section{DISCUSSION}

HAL are usually diagnosed by immunophenotyping, although most of cases are involved in abnormalities of chromosomes [8]. During proliferation and differentiation, the malignant leukemic cells not only present abnormal immunophenotypes, but also develop aberrant ultrastructural features [9]. The morphological characteristics in normal lymphocyte and myelocyte blast are so disparate that they can be discriminated by light microscopy and TEM. On other hand, the biphenotypic cells in HAL that express both lineage antigens do not always share typical ultrastructural features and are usually difficult to distinguish. Most of them meet the standard morphologic and ultrastructural criteria of ALL blasts, such as high nucleus-cell ratio, more heterochromatin, irregular nuclear outlines with incisures or notches, swollen mitochondria, and less of a Golgi apparatus, all of which hint at ALL [10].

In this studied group, the majority of blasts in 8 out of 15 cases $(53 \%)$ exhibited a strong lymphoid appearance: of them, 5 cases were explained as ALL, and the other 3 cases judged as biphenotypic HAL additionally showed some myeloid clues, were positive for MPO, and had moderate RER and Golgi bodies simultaneously. Biphenotypic cells may show monocytoid ultrastructures, abundant cytoplasm, twisted nuclei, moderate Golgi bodies, and, occasionally, cytoplasmic processes. Two cases (13\%) shared these points and were diagnosed as M5 in the group. The incidence of biclonal type with mixed lymphoid and myeloid cells was lower-2 cases $(13 \%)$ in the present study. In addition, a few cases had atypical lymphoid ultrastuctural characteristics, such that it was difficult to give a diagnosis given that they were MPO positive. Two cases in the study group $(13 \%)$ fell into this category and therefore were suspected of being HAL and one as M4. MPO positivity was an important fact or in the diagnosis of HAL. There were 7 out of 15 cases positive for MPO (46\%), besides a little myeloid feature, that were diagnosed or suggested as HAL by TEM. The MPO activity under electron microscopy was not consistent with flow cytometry analysis in a few cases, which might result from a different way of blast calculating blasts or sensitivity in different processes. In actual clinical test, MPO positivity from either of both results should be stressed.

\section{CONCLUSION}

HAL was easily recognized and diagnosed by TEM when cell components included those of both lymphoid and myeloid blasts. Most of biophinotypic leukemia cells of HAL had the lymphoid features ultrastructurally, though a few of them showed varied appearances. MPO reaction could mislead the investigator into a diagnosis of acute myeloid leukemia, but it was very an useful marker in diagnosis of HAL when malignant cells also demonstrated several lymphoid ultrastructural features. The great challenge of HAL diagnosis by EM is the differentiation of BAL from ALL when blasts are negative for MPO, as well as distinction from monoplastic leukemia (M5).

\section{REFERENCES}

1. Altman AJ. Clinical features and biological implications of acute mixed lineage (hybrid) leukemias. Am J Pediatr Hematol Oncol. 1990;12:123-133.

2. Matutes E, Morilla R, Farahat N, et al. Definition of acute biphenotypic leukemia. Haematologica. 1997;82:64-66.

3. Bene MC, Castoldi G, Knapp W, et al. Proposals for the immunological classification of acute leukemias. European Group for the Immunological Characterization of Leukemias (EGIL). Leukemia. 1995;9:17831786.

4. Francis C, Connelly MC. Rapid single-step method for flow cytometric detection of surface and intracellular antigens using whole blood. Cytometry. 1996;25:58-70.

5. Pontvert-Delucq S, Breton-Gorius J, Schmitt C, et al. Characterization and functional analysis of adult human bone marrow cell subsets in relation to B-lymphoid development. Blood. 1993;15;82:417-429. 
6. Roels F, Wisse E, Brest BDE, Meulen J. Cytochemical discrimination between catalases and peroxidases using diaminobenzidine. Histochemistry. 1975;41:281-312.

7. Buccheri V, Shetty V, Yoshida N, Morilla R, Matutes E, Catovsky D. The role of an anti-myeloperoxidase antibody in the diagnosis and classification of acute leukaemia: a comparison with light and electron microscopy cytochemistry. Br J Haematol. 1992;80: 62-68.

8. Carbonell F, Swansbury J, Min T, et al. Cytogenetic findings in acute biphenotypic leukaemia. Leukemia. 1996;10:1283-1287.

9. Henderson ES, Lister TA, Greaves MF. Leukemia. Peiking: Health Science Asia, Elsevier Science; 2002. 